\title{
ECMO for a Cardiac Arrest and Prolonged CPR in the Cath-Lab: Dose it worth? Case Report and Review of Literature
} \author{
Sadek ${ }^{1,4}$ \\ ${ }^{1}$ Prince Abdullah Bin Abdulaziz Bin Musaed Cardiac Center, Arar city, Saudi Arabia \\ ${ }^{2}$ Department of Cardiothoracic Surgery, Benha University Hospitals, Egypt \\ ${ }^{3}$ Ahmed Gasim Cardiac Center, Omdurman Islamic University, Sudan \\ ${ }^{4}$ Heart and diabetic center, Bad Oeynhausen, Germany
}

Mohamed Alassal ${ }^{1,2 *}$, Mohamed Saed Khalifa ${ }^{1,4}$, Mohamed Aldahmashi ${ }^{1}$, Saif Eldin Ibrahim ${ }^{1}$ and Marwan

Submission: February 21, 2017; Published: April 19, 2017

*Corresponding author: Mohamed Abdulwahab Alassal, Associate Professor and Consultant of Cardiothoracic surgery, Benha University

Hospitals, Egypt, Email: dmohamedabdelwahab@gmail.com

Abstract

Introduction: Nowadays the vast expansion to ECMO indications raises ethical questions such as whose patient should be treated with ECMO and when the ECMO support should be discontinued?

Case presentation: 57yr old gentleman presented as ACS and 1yr PCI was planned. CAG revealed total occlusion of LAD; LCX: mild lesions, RCA: totally occluded. Attempt to open the RCA was failed, LAD was stented but after that he got CP arrest. CPR was initiated and continued for 1.5h, then he revived, but echocardiography showed EF $10 \%$.

VA-ECMO was inserted then shifted to CCU and started to be awake. CXR showed massive left pleural effusion so exploratory thoracotomy was done which revealed big blood collection from intercostal artery and fracture multiple ribs.

In $3^{\text {rd }}$ day he was taken to the CathLab electively for another trial to open the RCA which was successfully done. ECMO was weaned off successfully in $5^{\text {th }}$ day post implantation. Echocardiography showed EF $40 \%$.

Then he got aggressive chest infection and septicemia. Hemodynamic deterioration increased so we re-implanted VA- ECMO again after $48 \mathrm{~h}$ from explantation for $2^{\text {nd }}$ time and he again started to improve.

In $3^{\text {rd }}$ week he got massive hematemesis, melena and upper GIT endoscopy revealed massive erosive gastritis. In $30^{\text {th }}$ day post cardiac arrest the oxygenator of the ECMO started to be clotted so ECMO was explanted and he deteriorated and death was declared in next day.

Conclusion: Earlier and rapid decision for ECMO is better and the question for starting ECMO in cardiogenic-shock before PCI needs to be raised and validated. Bleeding is the major risk of ECMO due to continuous infusion of heparin to protect ECMO circuit.

Keywords: ECMO; Cardiogenic shock; Cardiopulmonary arrest; CPR

\section{Introduction and Background}

Over the last several decades the scope of applications of mechanical ventricular assisting devices has widened, and the availability of easily deployable devices has increased significantly [1]. The rapid adoptions of new temporary devices necessitate physicians to become familiar with these commonly used technologies [2].
Despite advances in coronary revascularization and widespread use of primary percutaneous interventions, cardiogenic shock complicating an acute ST-elevation myocardial infarction remains a clinical challenge with high mortality rates [3]. Conservative management with catecholamines is associated with serious limitations, including 
arrhythmias, increased myocardial oxygen consumption, and inadequate circulatory support [4,5]. Clinicians have therefore turned to mechanical means of circulatory support. Circulatory assist systems for cardiogenic shock complicating an acute STelevation myocardial infarction can be distinguished by the method of placement (i.e. percutaneous vs. surgical), the type of circulatory support (i.e. left ventricular, right ventricular, or biventricular), and whether they are combined with extracorporal membrane oxygenation (ECMO) [6].

Extra Corporeal Membrane Oxygenation (ECMO) has remarkably progressed over the recent years and became a valuable tool in the care of patients with severe cardiac and pulmonary dysfunction refractory to conventional management [7]. Nowadays ECMO has become more reliable with improvement in equipment, and increased experience, which is reflected in improving results. The indications are extended to more prolonged use in intensive care unit, such as bridge to recovery, bridge to bridge or bridge to transplant, for both cardiac and lung transplant and support for complex cardiac and pulmonary procedures. The vast expansion to its indications raises ethical questions such as whose patient should be treated with ECMO and when the ECMO support should be discontinued $[7,8]$.

ECMO should only by performed by clinicians with good training and experience. ECMO is a supportive therapy rather than disease modifying treatment in itself; and the best results are obtained if we chose the right patient, the right type of ECMO and the right type of configuration (i.e. site, management and complication anticipation) [9].

In this report we present our case who got a cardiac arrest during primary PCI which necessitated prolonged CPR then VA-ECMO deployment.

\section{Case Presentation}

Our case is 57 years old gentleman, chronic heavy smoker, recently discovered type 2 Diabetes Mellitus (DM), hyperlipidemic, hypertensive and negative family history of ischemic heart diseases. He presented to Emergency Room (ER) with intermittent typical chest pain, associated with sweating in the last 12 hours. Echocardiography showed ejection fraction EF around 35\% with multiple regional wall motion abnormalities. He was hemodynamically stable but has dynamic ECG changes; he was managed as a case of acute coronary syndrome (ACS) and received a conventional treatment and planned for urgent PCI next day. In the ward he remained stable and asymptomatic for 6 hours after that he experienced chest pain with new LBBB so he was shifted to the Cath-lab for primary PCI. Coronary angiography revealed proximal total occlusion of LAD; big ramus coronary artery with tight long proximal lesion, LCX has non-flow limiting lesions, RCA totally occluded as well with communicating collaterals between LAD and some septal branches and RCA. Attempt to open the RCA was failed, so LAD was tried and the lesion was crossed, ballooned and stented with small caliber artery distal to the stent just after deployment of the stent, the patient went into pulmonary edema, hypotension and bradycardia then cardiopulmonary arrest. So cardiopulmonary resuscitation (CPR) was initiated and intra aortic balloon counter-pulsation (IABP) was inserted and temporary pace maker (TPM) was placed and the patient was intubated and mechanically ventilated. CPR was continuous for about one and half hours, during this period Re-coronary angiography was performed. The LAD was still open but the Ramus artery was sub-totally occluded which was re-opened and stented successfully with TIMI-3 flow. After that the patient revived but was on high inotropic support and IABP, and echocardiography showed EF around $10 \%$. So the consensus of the cardiology and cardiac surgery team was to place the patient on percutaneous VAECMO and to give him the best chance for possible survival. The family has been informed that the ECMO is the last resort and if unsuccessful there are no other treatment options.

VA-ECMO was deployed in the left groin in the Cath-Lab then the patient shifted to CCU and we could able to come down with the inotropes and the patient started to be awake and moving all limbs. In the first night the CVP was increased up to $26 \mathrm{~mm} \mathrm{H}_{2} \mathrm{O}$ and $\mathrm{CXR}$ showed massive left pleural effusion so left chest drain was inserted and more than 2 liters of bloody effusion was drained after that the CVP went down to $11 \mathrm{~mm}$ $\mathrm{H}_{2} \mathrm{O}$ and the ABG improved but repeated Chest X-Ray revealed recollection again in left pleura and his $\mathrm{Hb}$ continued dropping despite of continuous blood transfusions so he was taken to OR for exploratory left thoracotomy which revealed big amount of blood clots and actively bleeding intercostal artery and fracture ribs due to prolonged CPR with external cardiac massage. After that he became stable again and $\mathrm{Hb}$ started to build up.

After stabilization of the patient and became generally better with good ABGs, and good hemodynamics he was taken again to the Cath-Lab electively for another trial to open the RCA which was succeeded this time and RCA was opened and stented. The LAD and ramus artery' stents were found to be patent with TIMI-3 flow. The patient was shifted again to CCU and ECMO weaning-off process started gradually which was successfully done in the $5^{\text {th }}$ day post implantation. Echocardiography showed EF of $40 \%$.

Post ECMO removal period was unremarkable and he remained stable until after 36 hours from ECMO removal the patient got sudden and rapid deterioration and he required higher inotropes. Echocardiography showed global severe hypokinesia and EF went down to be about $15 \%$, so the decision was to take him for emergency coronary angiography which revealed that all coronary stents were occluded with fresh thrombi, so percutaneous suction thrombectomy was done from the three vessels (LAD, RCA and Ramus coronary artery) 
and IAB was inserted again in the Cath-lab. After that his hemodynamic profile improved. Then we started to decrease sedations to start weaning from the ventilator which failed due to severe chest infection (ventilator acquired pneumonia VAP) and flail chest due to bilateral multiple ribs and sternum fractures after previous CPR, so bed-side percutaneous tracheostomy was done.

The patient got progressive and aggressive infection with +ve blood cultures and +ve sputum cultures as well (acintobacter boumani, and pseudomonas carnii). Also he required renal hemodialysis CRRT due to deteriorating kidney function and high creatinine. Then he got a septic shock with Hemodynamic deterioration and required more inotropes again so after consensus the decision was to place him on $2^{\text {nd }}$ time VA-ECMO again. Percutaneous left femoral VA-ECMO was inserted for $2^{\text {nd }}$ time and the patient again started to improve and Echocardiography showed an improvement in the cardiac contractility as well with EF about 35\%.

After few days the left leg (same side of ECMO) started to be cold and pale despite there is shunt connection from the arterial line to the femoral artery to supply the leg distal to arterial cannula insertion. Vascular angiography revealed clotted tibialis anterior artery so the ECMO was shifted to the right side and open embolectomy to left tibialis anterior artery was done using Fogarty catheter and the blood flow was good again and the color of the leg improved and became warm but the distal half of the foot did not improve and became cyanotic and the process of dry gangrene started in the tips of the left toes.

Trials of weaning from ECMO were done frequently but were failed although he remained hemodynamically stable until the $3^{\text {rd }}$ week post cardiac arrest, the patient got massive hematemesis and melena so, upper GIT endoscopy was done and revealed massive erosive gastritis. In the $30^{\text {th }}$ day post cardiac arrest the oxygenator of the ECMO started to give poor oxygination then started to be clotted so the decision was to explant the ECMO device. After that the patient deteriorated more and death was declared in next day from ECMO removal

\section{Discussion}

The percutaneous assist systems most commonly used in cardiogenic shock MI are the intra-aortic balloon pump (IABP), venoarterial ECMO, the Impella pump, and the Tandem-Heart.

Recently, the large randomized IABP-Shock II Trial did not show a significant reduction in 30-day mortality in CSMI with IABP insertion $[10,11]$. There are no randomized study data available for ECMO use in CSMI. Both the Impella pump and the Tandem-Heart did not reduce 30-day mortality when compared with IABP in small randomized controlled trials (RCTs). Despite the need for effective mechanical circulatory support in CSMI, current devices, as tested, have not been demonstrated to improve short- or long-term survival rates [12].

A meta-analysis by Cheng et al. [12] of 1,866 patients receiving ECMO for treatment of cardiogenic shock and cardiac arrest showed a survival to hospital discharge between 20.8$65.4 \%[12,13]$.

Schmidt et al. [13] developed a survival calculator based on analysis of 3,846 patients with refractory cardiogenic shock treated with ECMO between 2003 and 2013 [14]. Improved survival rates are associated with treatment of patients with cardiogenic shock from myocarditis, refractory VT/VF or post heart or lung transplantation. Other factors such as decreased age significantly improve predicted survival particularly for patients less than 63 years of age $[14,15]$ and this was one of the reasons to deploy the ECMO machine in our patient despite of long time CPR because he was relatively young ( 57 years).

However, cardiogenic shock is not only a decrease in cardiac contractile function, but also a multiorgan dysfunction syndrome(MODS) resulting from peripheral hypoperfusion with microcirculatory dysfunction, often complicated by a systemic inflammatory response syndrome (SIRS) and sepsis. Once MODS has developed, it is difficult to improve prognosis and reduce mortality by simply increasing cardiac output with a circulatory assist device [15], and this exactly what happened in our case as it was complicated with sepsis and MODS as well. Prevention of MODS may depend on three critical factors:

a) Optimal timing (i.e. early initiation) of mechanical circulatory support.

b) Optimal level of mechanical circulatory support with reestablishment of adequate perfusion of critical organs.

c) Optimal prevention and management of potential device-related complications (i.e. device malfunction, infection) $[15,16]$.

We have learned a lesson from this case that we must call for ECMO in cases post witnessed cardiopulmonary arrest within 10 minutes after the beginning of CPR, so we can put the patient on ECMO earlier in a suitable time. We must put in consideration that within 60 minutes from an effective CPR the patient should be put on the ECMO machine (including CPR+ECMO deployment time) and in our instate, we (as a team from cardiac surgery, the cardiology, ER and anesthesia department) have written an internal policy and protocol for ECMO use including: post CPR in Cath-lab, in ER or any department in the hospital and we initiated an "ECMO code team" to be ready all the time "24\7" for any emergency once it is indicated.

We confronted in the beginning with misconceptions and they are common. Majority of our colleagues in other departments "fear" the ECMO patient and misunderstand the 
roles of the team members involved in care of these patients. The "fear" comes from ignorance and lack of experience and leads to potential delays in referral and subsequent care and this was what happen exactly in our case as the cath-lab team consulted us for VA-ECMO lately after long time from the beginning of CPR and this delay have worsened the outcome.

\section{Conclusion and Take Home Message}

Earlier and rapid decision for ECMO insertion is better and the question for starting ECMO in Cardiogenic shock before PCI needs to be raised and validated for better patient survival and good results. Collaboration between different hospital departments must be there and is mandatory to build up efficient ECMO service which can meet the needs for these critically ill patients.

Bleeding is the major risk of the ECMO due to continuous infusion of heparin to protect the ECMO circuit from clotting and as in this case the balance between the risk of stent thrombosis and bleeding was delicate with initial stent thrombosis as a price to stop massive bleeding in the pleural space and the GIT bleeding and erosive gastiritis as a price of for keeping patent stents and ECMO circuits. CPR although life saving but may result in rib fracture and continued bleeding after ECMO which needs more attention to be done gently as possible.

While ECMO may not be an optimal solution, it does increase the survival in certain patient populations compared to conventional treatment. "Code ECMO" team should be initiated from a cardiac surgery team and a perfusionist and should be ready $24 \backslash 7$ to place the indicated patient on ECMO for any respiratory or cardiac or cardiopulmonary failure.

\section{References}

1. Borisenko O, Wylie G, Payne J, Bjessmo S, Smith J, et al. (2014) The cost impact of short-term ventricular assist devices and extracorporeal life support systems therapies on the National Health Service in the UK. Interact Cardiovasc Thorac Surg 19(1): 41-48.

2. Sauer CM, Yuh DD, Bonde P (2015) Extracorporeal membrane oxygenation use has increased by $433 \%$ in adults in the United States from 2006 to 2011. ASAIO J 61(1): 31-36.

3. Chamogeorgakis T, Rafael A, Shafii AE, Nagpal D, Pokersnik JA, et al. (2013) Which is better: a miniaturized percutaneous ventricular assist device or extracorporeal membrane oxygenation for patients with cardiogenic shock? ASAIO J 59(6): 607-611.
4. Wu MY, Lin PJ, Lee MY, Tsai FC, Chu JJ, et al. (2010) Using extracorporeal life support to resuscitate adult postcardiotomy cardiogenic shock. Treatment strategies and predictors of short-term and mid-term survival. Resuscitation 81(9): 1111-1116.

5. Ouweneel DM, Schotborgh JV, Limpens J, Sjauw KD, Engström AE, et al. (2016) Extracorporeal life support during cardiac arrest and cardiogenic shock: a systematic review and meta-analysis. Intensive Care Med 42(12): 1922-1934.

6. Abnousi F, Yong CM, Fearon W, Banerjee D (2015) The Evolution of Temporary Percutaneous Mechanical Circulatory Support Devices: a Review of the Options and Evidence in Cardiogenic Shock. Curr Cardiol Rep 17(6): 40

7. Werdan K, Gielen S, Ebelt H, Hochman JS (2014) Mechanical circulatory support in cardiogenic shock. Eur Heart J 35(3): 156-167.

8. Saffarzadeh A, Bonde P (2015) Options for temporary mechanical circulatory support. J Thorac Dis 7(12): 2102-2111.

9. Moll V, Teo EY, Grenda DS, Powell CD, Connor MJ, et al. (2016) Rapid Development and Implementation of an ECMO Program. ASAIO J 62(3): 354-358.

10. Karagiannidis C, Brodie D, Strassmann S, Stoelben E, Philipp A, et al. (2016) Extracorporeal membrane oxygenation: evolving epidemiology and mortality. Intensive Care Med 42(5): 889-896.

11. Siao FY, Chiu CC, Chiu CW, Chen YC, Chen YL, et al. (2015) Managing cardiac arrest with refractory ventricular fibrillation in the emergency department: conventional cardiopulmonary resuscitation versus extracorporeal cardiopulmonary resuscitation. Resuscitation 92: 7076.

12. Sakamoto T, Morimura N, Nagao K, Asai Y, Yokota H, et al. (2014) Extracorporeal cardiopulmonary resuscitation versus conventional cardiopulmonary resuscitation in adults with out-of hospital cardiac arrest: a prospective observational study. Resuscitation 85(6): 762768.

13. Cheng R, Hachamovitch R, Kittleson M, Patel J, Arabia F, et al. (2014) Complications of extracorporeal membrane oxygenation for treatment of cardiogenic shock and cardiac arrest: a meta-analysis of 1,866 adult patients. Ann Thorac Surg 97(2): 610-616.

14. Schmidt M, Burrell A, Roberts L, Bailey M, Sheldrake J, et al. (2015) Predicting survival after ECMO for refractory cardiogenic shock: the survival after veno-arterial-ECMO (SAVE)-score. Eur Heart J 36(33): 2246-2256.

15. Sattler S, Khaladj N, Zaruba MM, Fischer M, Hausleiter J, et al. (2014) Extracorporal life support (ECLS) in acute ischaemic cardiogenic shock. Int J Clin Pract 68(4): 529-531.

16. Blumenstein J, Leick J, Liebetrau C, Kempfert J, Gaede L, et al. (2015) Extracorporeal life support in cardiovascular patients with observed refractory in-hospital cardiac arrest is associated with favourable short and long-term outcomes: a propensity-matched analysis. Eur Heart J Acute Cardiovasc Care 5(7): 13-22. 
Your next submission with Juniper Publishers will reach you the below assets

- Quality Editorial service

- Swift Peer Review

- Reprints availability

- E-prints Service

- Manuscript Podcast for convenient understanding

- Global attainment for your research

- Manuscript accessibility in different formats

( Pdf, E-pub, Full Text, Audio)

- Unceasing customer service

Track the below URL for one-step submission https://juniperpublishers.com/online-submission.php 